\title{
Kann der Staat nachhaltiges Konsumverhalten steuern?
}

\author{
Verbraucheraufklärung, freiwillige Vereinbarungen, Ordnungsrecht etc. - wel- \\ che Instrumente und Maßnahmen motivieren die Masse der VerbraucherInnen zu \\ nachhaltigem Konsum? Und welche Perspektiven ergeben sich für die Politik? \\ Eine empirische Untersuchung von typischen Konsumtrends in den letzten 20 \\ Jahren sowie eine Befragung von PraxisakteurInnen ergab, dass vor allem sy- \\ stemische Ansätze bei Produkten und Politikstil gefordert sind. Vertieft be- \\ trachtet wurden die Bereiche Waschen, Ernährung und Heizen (1).
}

Von Ulla Simshäuser

ber die Vertiefungsbereiche hinweg lassen sich allgemeine Wirkungen von Instrumenten feststellen, die sich in folgenden Trends ausdrücken:

- Die bisher eingesetzten Instrumente trugen im Rahmen eines jeweils bereichsspezifischen Mixes zum Teil sehr wirksam zu einer Verbesserung der Produkteffizienz bei;

die Einflüsse der Umwelt- und Verbraucherberatungen auf eine Veränderung des Verbraucherverhaltens breiter Konsumentengruppen sind moderat;

auf lange Sicht ist derzeit keine Beeinflussung gegenläufiger Wachstumseffekte zu beobachten.

Die einzelnen Instrumente hatten dabei jeweils unterschiedliche und über den einzelnen Vertiefungsbereich hinaus nicht verallgemeinerbare Wirkungen. Beispielsweise verzeichnete das Umweltzeichen unterschiedliche Erfolge.

Für die Zukunft zeichnen sich deshalb neue Anforderungen an die Produktpolitik und den Politikstil für nachhaltigen Konsum ab: Im Fokus stehen systemische Ansätze im Bereich der Produkte, sogenannte systemische Innovationen, wie auch im Politikstil, hier durch Vernetzung von Akteuren und Handlungsfeldern.

\section{- Begrenzte Beeinflussung durch Verbraucherinformation}

Bisher ging man davon aus, dass Verbraucherverhalten in erster Linie durch Information und Beratung beeinflusst werden könne. Unsere Untersuchungen können dies mit dem Blick auf die Masse der VerbraucherInnen nicht bestätigen. Insbesondere finanzielle und zeitliche Faktoren, Gegebenheiten der Infrastruktur sowie nicht zuletzt grundsätzliche Wert- haltungen wie sie sich im Rahmen individueller Lebensstile entwickeln, scheinen verhaltensbestimmend zu sein. Materielle Anreize scheinen indes für die Mehrzahl der VerbraucherInnen über Ansätzen der Bildung und Information zu stehen. Auch Instrumente, die das wirtschaftliche Eigeninteresse der VerbraucherInnen ansprachen, wie etwa im Bereich Heizkosten, wurden relativ leicht entscheidungs- und verhaltenswirksam. Der Lenkungsanreiz, der von ihnen ausgeht, ist allerdings angesichts des hohen allgemeinen Wohlstandsniveaus für viele Menschen relativ gering.

\section{Zielkommunikation und Wir- kungsdynamik als Potenziale}

An verschiedenen Beispielen zeigte sich, dass weniger ein einzelnes, auf das Verbraucherverhalten zielendes Instrument als solches wirksam war, sondern die mit seiner Entwicklung und Anwendung verbundene soziale Dynamik. Nicht in dem ursprünglich direkt intendierten Steuerungsimpuls als solchem, sondern in der mitunter nicht intendierten gesellschaftlichen Dynamik, die sich häufig bereits vor der eigentlichen Maßnahme entwickelte, liegen offenbar die eigentlichen Potenziale von Instrumenten. Instrumente werden als Anlass zum Aushandeln und Kommunizieren von Zielen benutzt. Die Besonderheit besteht aber darin, dass die entstehende Dynamik rund um die Zielkommunikation nach mehreren Seiten wirkt und verschiedene Akteure beeinflusst.

Charakteristisch für diese Dynamik ist zum Beispiel die beobachtete widersprüchliche Wirkung von Maßnahmen der Verbraucherinformation und -beratung. Einerseits war ihre direkte Wirkung auf Verhaltensänderungen der Gesamtheit der VerbraucherInnen relativ gering. Andererseits setzte die beratende Tätigkeit der Verbraucherorganisationen - Produkttests, Labels und dergleichen eingeschlossen - Signale an andere Akteure über mögliche künftige Konsumtrends und veranlasste Hersteller zu ökologisch relevanten Produktentwicklungen. Ähnliches pro-aktives Handeln lösten Diskussionen staatlicher Akteure und Verbraucherorganisationen über Inhaltsstoffe in Waschmitteln und den Versuch einer ordnungsrechtlichen Regelung aus. Dort, wo in den untersuchten Vertiefungsbereichen der Instrumentenmix erfolgreich war, war vor allem die Dynamik entscheidend, die sich rund um den Instrumenteneinsatz entwickelte. Das rückt die Bedeutung des Politikstils, also die Art der Verständigung der Akteure vor und nach dem Instrumenteneinsatz, in den Mittelpunkt. Normative, an irreversiblen Standards orientierte Instrumente scheinen in manchen Bereichen der Entwicklung nicht Rechnung zu tragen und drohen Akzeptanzverluste zu erleiden, bei manchen Produkten gilt dies auch für den Blauen Engel.

\section{- Ein Steuerungspradoxon}

Wie die Konsum- und Lebensstilforschung zeigt, wurden mit dem gesellschaftlichen Wandel seit den 80-er Jahren sowohl die Lebensstile als auch die für den nachhaltigen Konsum relevanten Akteure - wie Umwelt- und Verbraucherorganisationen, Hersteller, Handelsunternehmen etc. zahlreicher. Zu vielen gesellschaftlichen Milieus hat der Staat inzwischen nur noch über die entsprechenden relevanten Akteure Zugang. Dies mündet in ein für den nachhaltigen Konsum typisches Steuerungsparadoxon des Staates. Die Vielfalt der Akteure und Lebensstile nimmt einerseits zu, so dass gerade bei der Frage der Verhaltensbeeinflussung akteurs- und zielgruppenspezifische Ansätze im Zugang auf diese Lebensstilgruppen notwendig sind. Gleichzeitig übersteigt gerade diese Vielfalt der Lebensstile von VerbraucherInnen die Möglichkeiten insbesondere des Staates zur Feinsteuerung. Zu lösen ist dieses Dilemma nur über einen Politikstil, der flexibel und in der Lage ist, die Handlungspotenziale verschiedener Akteure einzubinden in eine gemeinsame Strategie und geteilte Ziele.

\section{Staatliche Doppelrolle}

Dem Staat kommt heute die besondere Aufgabe zu, relevante Akteure zu identifizieren und ihre Handlungspotenziale zu einer effizienten Strategie zu vernetzen. Er hat moderierende Aufgaben wie:

das Formulieren, Verbreiten und Inszenieren von geeigneten Zielen des nachhaltigen Konsums 
und deren Umsetzung, zum Beispiel durch Öffentlichkeitsarbeit, Kampagnen, Marketingförderung und das Unterstïtzen von Akteursnetzwerken;

das Fördern der Eigeninitiative relevanter Innovations-Akteure im Bereich des nachhaltigen Konsums und das Erleichtern ihres Marktzugangs.

So demokratisch diese Strategie daherkommt, so risikoreich dürfte sie in der Praxis sein. Die Gefahr besteht, dass die Partikularinteressen von Akteuren in politische Ziele gegossen werden. Gerade wegen des am Konsens der Akteure orientierten Politikstils kommt dem Staat notwendigerweise wieder eine viel stärkere und bei weitem nicht ausgeschöpfte Verantwortung für die Gefahrenprävention zu. Jenseits einer einseitigen Verstrickung in Grenzwertdebatten und eines reinen Reagierens auf immer schnellere Veränderungen von Techniken und Standards gilt: Staatliche Akteure müssen definieren, wo die Suche nach Konsens aufhört, und verständlich und breitenwirksam kommunizieren, wo reale Risiken bestehen und wo das Prinzip der politischen Verantwortung, gerade auch für künftige Generationen, verbindlich eingehalten werden und mit Inhalt gefüllt werden muss. Dazu gehört neben dem Schutz der natïrlichen Ressourcen insbesondere der gesundheitsbezogene Schutz der VerbraucherInnen vor Gefahren im Umgang mit Stoffen, Produkten, Lebensmitteln und Techniken.

\section{Systemische Innovationen fördern}

Angesichts der begrenzten Erfolge verhaltensbeeinflussender Instrumente zur Förderung von nachhaltigem Konsum und steigender Wachstumstrends sollte die konsequente Entwicklung und Förderung derjenigen Produktinnovationen vorangetrieben werden, die über eine reine Orientierung an Effizienzstandards für bestehende Produkte hinaus gehen. Angesprochen sind technische Innovationen im Produktbereich, die etwa das Ziel der Null-Emission in nahezu geschlossenen Kreisläufen anstreben oder auch technische Lösungen, die schwer zu beeinflussendes Routinehandeln der VerbraucherInnen im Alltag ausgleichen. Ein Beispiel ist hier die automatische Dosierung von Waschmitteln. Ökologischem Produktdesign und Integrierter Produktpolitik kommt deshalb eine entsprechende Verantwortung zu. Künftige Orientierungen müssten zumindest für bestimmte Marktsegmente und Konsumentengruppen auch eine Verlagerung von Produkt- zu Dienstleistungskonsum anstreben und Strategien zur Unterstiutzung der dafür notwendigen Infrastruktur entwickeln. Hierzu bedarf es auch neuartiger unterstïtzender Maßnahmen und flexibler Instrumente.

\section{Monitoringmodelle entwickeln}

Dies stellt auch die anwendungsorientierte Forschung vor neue Herausforderungen. Wenn sowohl für die Entwicklung neuer Produkte als auch für die Entwicklung neuer Instrumente und des Politikstils systemische Ansätze favorisiert werden, muss die angewandte Forschung sich durch neue prozessorientierte Methoden entsprechend anpassen. Hierzu gehört, auch das ist ein Ergebnis des Forschungsprojekts, dass klassische Input-Output-Evaluationen für die praxisorientierte Politikberatung künftig eher in den Hintergrund treten und prozess- und projektbegleitenden Monitoringmodellen Platz machen müssen.

\section{Anmerkung}

(1) Das umfangreiche Forschungsprojekt wurde vom IÖW unter dem Titel: „Evaluation und Optimierung von Instrumenten zur Förderung von nachhaltigem Konsum" von 1997-2000 im Auftrag des Umweltbundesamtesdurchgeführt. Es war Teil eines umfassenden Demonstrationsvorhabens mit mehreren Teilprojekten, dessen jeweilige Ergebnisse bereits auf der zweiten Tutzinger Tagung zum nachhaltigen Konsum im April 2000 vorgestellt wurden. Vgl. auch den Abschlussbericht Hagemann, Helmut/Simshäuser, Ulla (unter Mitarbeit von Frieder Rubik und Gerd Scholl): Instrumente zur Förderung von nachhaltigem Konsum - Evaluation und Optimierung. IÖW, Heidelberg. Eine Veröffentlichung ist geplant.

Die Autorin
Dr. Ulla Simshäuser ist wissenschaftliche Mitarbeiterin
des Institut für ökologische Wirtschaftsforschung.
Kontakt: IÖW-Regionalbüro Baden Württemberg,
Bergstr. 7, 69120 Heidelberg, Tel. 06221/ 64916-7, E-
mail: mailbox@heidelberg.ioew.de

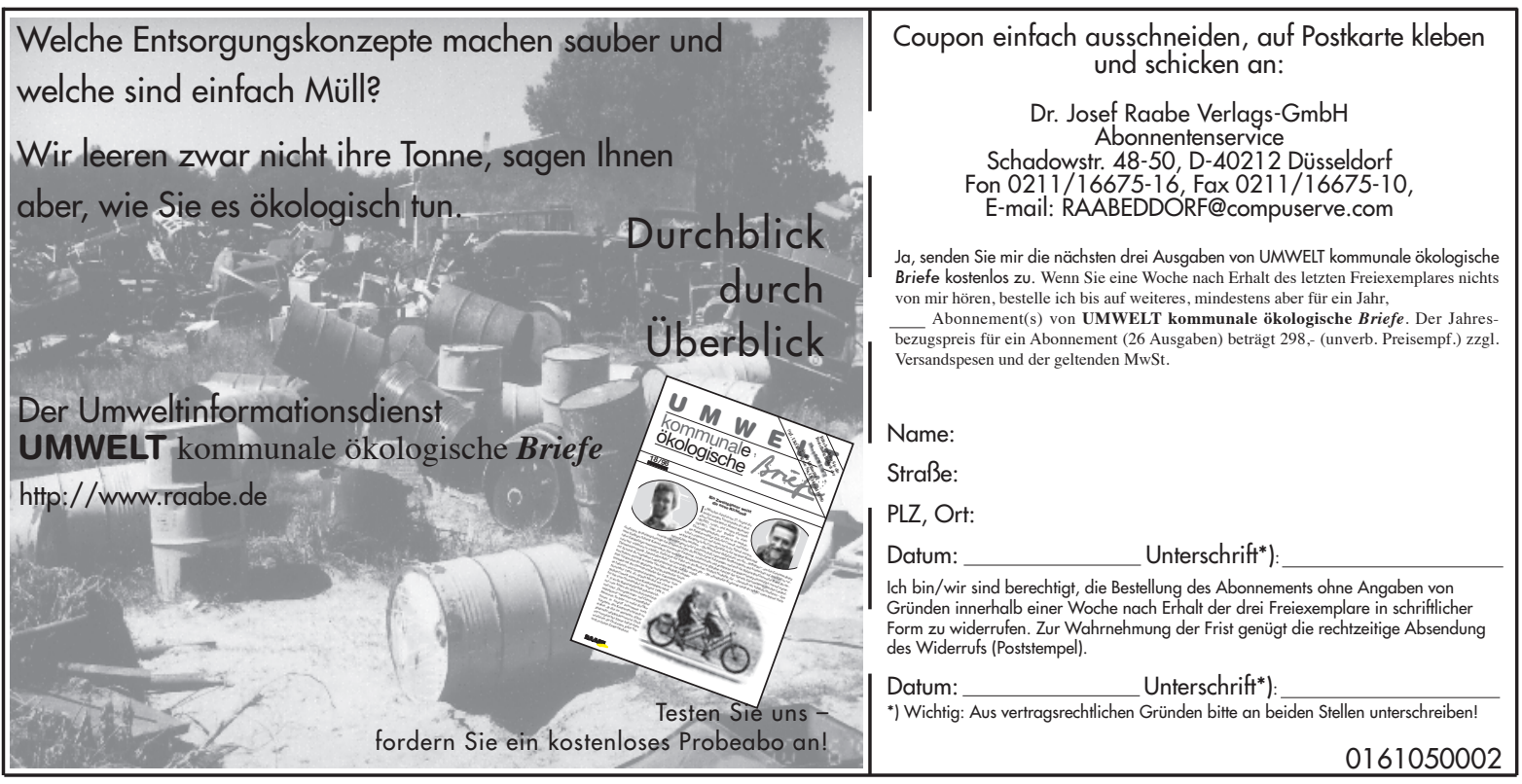


(c) 20I0 Authors; licensee IÖW and oekom verlag. This is an article distributed under the terms of the Creative Commons Attribution Non-Commercial No Derivates License (http://creativecommons.org/licenses/by-nc-nd/3.o/), which permits unrestricted use, distribution, and reproduction in any medium, provided the original work is properly cited. 